\title{
AN E-TUTOR TOOL FOR GRAMMATICAL ERROR CORRECTION
}

\author{
Xiaodong Sun, Yanqin Yin, Huanhuan Lv, Pikun Wang, Hongwei Ma and Dongqiang Yang* \\ School of Computer Science and Technology, Shandong Jianzhu University, Jinan 250101, China
}

\begin{abstract}
Intelligent language learning tools have evolved into an inevitable aid for ESL/EFL (English as second or foreign language) learners to improve their linguistic skills. By functioning as a machine translation task, automatic grammatical error correction (GEC) has made significant progress with the help of deep neural networks, but its accuracy and coverage rates on different error types have not been fully satisfactory in practice. This paper designs an e-Tutor tool for GEC to help ESL/EFL learners automatically inspect and correct their grammatical errors in writing. One of the core research tasks in GEC is how to improve its generalizability while dealing with more complex error types. In the paper, we propose a novel data augmentation method to add artificial noise to native English corpora during training a neural translation model for GEC. To improve the output quality of GEC, we also design a re-editing module, which mainly consists of a statistical language model, along with a grammatical error detection classifier, in validating each sentence generated by GEC. Experiment results on GEC show that our e-Tutor tool can achieve state-of-the-art performance on the CoNLL-2014 dataset.
\end{abstract}

\section{KEYWORDS}

e-Tutor Tool, Data Augmentation, Grammatical Error Correction

\section{INTRODUCTION}

The goal of GEC is to take learners' erroneous sentences as input, translating or converting them into grammatically correct ones. Early research methods on GEC were based on grammatical rules and linguistic statistics. For example, Gamon et al. (2008) used a rule-based n-gram language model in correcting prepositions and qualifiers errors for ESL/EFL learners. Junczys-Brockett et al. (2006) proposed to adopt the statistical machine translation model into the GEC task, and their method was to automatically translate erroneous sentences into the correct ones that can satisfy the grammatical requirements for native English. Current studies usually take GEC as a neural machine translation (NMT) process and focus on architectural design of deep neural networks to realize the NMT's encoder-decoder pipeline.

In spite of recent breakthroughs in deep language processing (Torfi et al., 2020), GEC is still a challenging task, partly due to the diversity of grammatical error types in learner language as ESL/EFL, and partly due to the complexity of interaction among multiple errors co-existing in context. Therefore, how to synthesize more learner data to feed and train a NMT model is key to enhance the generalizability of GEC while dealing with new or complex errors. To solve the problem of data scarcity on manually annotated learner corpora, Kiyono et al. (2019) proposed a data augmentation method that pre-trained a NMT model using artificially generated learner data. Katsumata and Komachi (2019) also showed that the training process of NMT required a large amount of learner data to deliver an effective GEC tool, and generating pseudo learner data can assist GEC to improve NMT's generalizability.

In the paper, we propose a novel method to automatically construct the learner dataset for training a NMT model for GEC. First, for a word (i.e., a verb or noun), we generate all of its inflected forms, which are then collected into its confusion set. The spelling candidate set, generated by the Aspell (Grundkiewicz et al., 2019), is also combined with the confusion set to jointly synthesize spurious training data. We also introduce a two-stage processing for GEC: after training a NMT-based module with the synthesized data, we conduct the first-round GEC with it; we run the second-round GEC with a rule and statistical language module. The hybrid use of multiple correction methods can significantly improve our e-Tutor's performance on GEC.

\footnotetext{
* Correspondence author, ydq@sdjzu.edu.cn
} 


\section{E-TUTOR DESIGN}

\subsection{Architecture}

Our e-Tutor architecture consists of three modules: pseudo data generation, NMT, and re-editing, as shown in Figure 1. Briefly, apart from pseudo data generation, the NMT-based GEC module has multiple layers of deep neural networks working as an encoder and decoder pipeline. Given the candidate sentences yielded from the NMT module, the re-editing module rectifies them with a rule-based punctuation checker and a language statistics-based misspelling corrector. Finally, a binary classifier on grammatical error detection selects an optimal sentence as output.

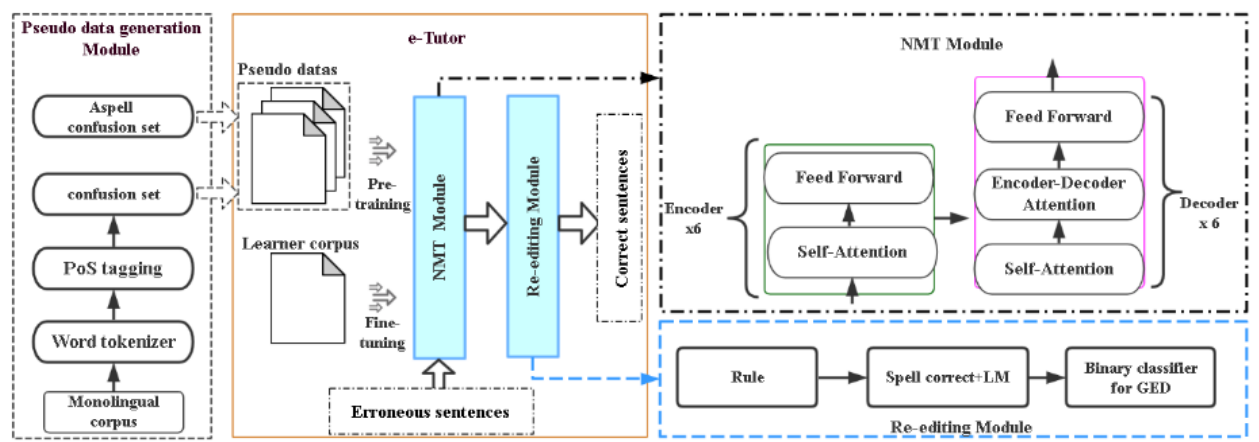

Figure 1. The e-Tutor workflow

\subsection{Generating Pseudo Data}

To improve the generalizability of NMT-based GEC, Grundkiewicz et al. (2019) suggested an unsupervised way of generating learner data. They first constructed a confusion set for a target word in a grammatically correct sentence using a spelling checker, and then replaced the target word with each word in the confusion set to produce spurious sentences for expanding the training data. Inspired by Grundkiewicz et al (2018), we design a novel way of data augmentation for GEC. Specifically, we first randomly select some words to introduce the token level of artificial errors, which consist of $15 \%$ of words in a native corpus. To improve the robustness of GEC on misspelling, one of the typical errors in ESL/EFL learners, we randomly conduct a character-level editing (i.e., insertion/deletion/replacement/swap) on $5 \%$ of words in a native corpus. The whole process of data augmentation is depicted as follows:

Rule 1: According to the established verb or noun transformation table, we first locate the inflected forms of a target word in a native sentence. We then select each of its inflected forms to replace it to form a learner sentence. If the target word has no inflection available, a candidate word in its spelling confusion set is randomly selected to replace it.

Rule 2: Randomly delete a word.

Rule 3: Randomly insert a word after a selected word.

Rule 4: Randomly swap with the next word.

Rule 5: Randomly change the case of the initial character in a word.

\subsection{Re-Editing}

To improve the correction quality of e-Tutor, we re-edit the candidate sentences, translated from the NMT-based GEC, with the following procedures:

1. A rule-based punctuation checker. The first letter of each sentence should be capitalized, and double punctuations and spaces should be removed.

2. Statistical spelling correction. For a target word, we first use Aspell (Grundkiewicz et al., 2019) to yield its spelling variants. After replacing the target with its variants to form a new cluster of candidate sentences, we run a N-gram Language Model (NLM) to rank them and output the sentence with the highest possibility. We calculate statistical information from corpora to estimate the distributional probability of 
n-grams. In the experiment, we used the Kenlm (Heafield, 2011) toolkit to train a $\mathrm{N}$-gram language model (i.e., $\mathrm{n}=6$ ) on the Gutenberg corpus (Lahiri, 2014).

3. Grammatical Error Detection (GED). We also train a binary classifier with BERT (Devlin et al., 2019) on the CoLA dataset (Warstadt et al., 2019) to assist error detection.

\section{EXPERIMENTS}

\subsection{Learner Corpus}

The existing learner corpora include FCE v2.1 (Dale and Kilgarriff, 2011), Lang8 (Mizumoto et al., 2011), NUCLE (Dahlmeier et al., 2013), and W\&i+LOCNESS (Granger, 2014; Bryant et al., 2019). The types of errors that ESL/EFL learners often make are related with spelling, nouns, subject-verb agreement, and verb forms, among the others. To accurately cover more error types, we also used NUCLE and W\&i+LOCNESS for fine-tuning NMT.

\subsection{Data Augmentation}

To generate pseudo learner data, we first preprocessed Google Billion Word Benchmark (Chelba et al., 2014), a public news corpus. After normalizing punctuations and limiting sentence length up to 80 words, we ran PoS tagging on the preprocessed corpus. For verbs and nouns, randomly selected, we replaced them with their respective inflection forms to produce learner data, as shown in Table 1. Since misspelling is one of the common grammatical mistakes, we also used Aspell to generate misspellings for a target word and stored them in its corresponding confusion set. Table 1 shows the inflection and misspelling confusion sets for a target word, after we conducted PoS tagging and lemmatization with NLTK and Aspell.

Table 1. Basic rules for generating a confusion set

\begin{tabular}{|c|c|c|c|}
\hline & Tag & Explanation & Inflection confusion set \\
\hline V & $\begin{array}{l}\text { VB } \\
\text { VBD } \\
\text { VBG } \\
\text { VBN } \\
\text { VBP } \\
\text { VBZ }\end{array}$ & $\begin{array}{l}\text { Verb, the base form } \\
\text { Verb, past tense } \\
\text { Verb, gerund or present participle } \\
\text { Verb, past participle } \\
\text { Verb, non-3rd person singular present } \\
\text { Verb, 3rd person singular present }\end{array}$ & accept: accepts, accepting, accepted \\
\hline $\mathrm{N}$ & $\begin{array}{c}\text { NN } \\
\text { NNS } \\
\text { NNP } \\
\text { NNPS }\end{array}$ & $\begin{array}{l}\text { Noun, singular or mass } \\
\text { Noun, plural } \\
\text { Proper noun, plural } \\
\text { Proper noun, plural }\end{array}$ & devote: devotion, devotions \\
\hline & & Word & Spelling confusion set \\
\hline & & $\begin{array}{l}\text { doing } \\
\text { future } \\
\text { have }\end{array}$ & $\begin{array}{l}\text { ding, dding, Dion, dingo, dingy } \\
\text { Futures, feature, suture, future's } \\
\text { Heave, Havel, haven, haves, gave }\end{array}$ \\
\hline
\end{tabular}

\subsection{Transformer Model}

The NMT in the paper mainly employs the Copy-Augmented Transformer (Zhao et al., 2019) model to build an encoder-decoder for GEC. The Copy-Augmented Transformer can determine whether to copy or generate a word in the correction process; only a few words in an erroneous sentence needs to be corrected, and most of the remaining ones are directly copied into the original positions in the sentence. The final probability distribution for a newly generated word is the weighted fusion of the decoding probability $p_{t}^{g e n}(w)$ and copy probability $p_{t}^{\text {copy }} . q_{t}$ is the mapping of the hidden state of decoding at time $t$. The keys and values are packed together into matrices $\mathrm{K}$ and $\mathrm{V} . K, V$ are different transformations of the hidden state of the input code. $W$ stands for the parameter matrix.

$$
P_{t}(w)=\left(1-\alpha_{t}^{\text {copy }}\right) * p_{t}^{g e n}(w)+\left(\alpha_{t}^{\text {copy }}\right) * p_{t}^{\text {copy }}(w)
$$




$$
\begin{gathered}
\alpha_{t}^{\text {copy }}=\operatorname{sigmoid}\left(W^{T} \sum\left(\left(q_{t}^{T} K\right)^{T} \cdot V\right)\right) \\
P_{t}^{\operatorname{copy}}(w)=\operatorname{softmax}\left(\left(q_{t}^{T} K\right)^{T}\right) \\
q_{t}, K, V=h_{t}^{\operatorname{trg}} W_{q}^{T}, H^{s r c} W_{k}^{T}, H^{s r c} W_{v}^{T}
\end{gathered}
$$

\subsection{Fine-Tuning}

To improve adaptability and recall rate of our Transformer-based GEC, which had been trained with our pseudo learner data, we combined the training datasets of NUCLE and W\&I+LOCNESS to fine-tune it. After that, the GEC outputs were ranked and re-edited with the rule and statistics-based correctors, together with the BERT-based GED to yield the final result.

Table 2. CoNLL-2014 test set results

\begin{tabular}{cccc}
\hline Model & \multicolumn{3}{c}{ CoNLL-2014 $\left(\boldsymbol{M}^{\mathbf{2}}\right.$ score $)$} \\
\hline $\begin{array}{c}\text { Chollampatt and Ng (2018) with Multilayer Convolution Neural Network } \\
\text { (4 ensemble models), Rescoring, Spell Check, Learner corpus }\end{array}$ & Pre. & Rec. & $\boldsymbol{F}_{\mathbf{0 . 5}}$ \\
Grundkiewicz and Junczys-Dowmunt (2018) with SMT, NMT, LM, & 65.5 & 33.1 & 54.8 \\
$\quad \begin{array}{c}\text { Learner corpus } \\
\text { Lichtarge } \text { et al. (2019) with Transformer, best Ensemble, Learner corpus, } \\
\text { Data augmentation }\end{array}$ & 66.8 & 34.5 & 56.3 \\
Commercial APP: Ginger API ${ }^{1}$ & 66.7 & 43.9 & 60.4 \\
\hline Our e-Tutor model after pre-training & 12.8 & 16.6 & 13.4 \\
\hline $\begin{array}{c}\text { plus fine-tuning } \\
\text { plus re-editing }\end{array}$ & 58.5 & 28.0 & 48.0 \\
& 70.4 & 39.6 & 60.9 \\
& $\mathbf{7 0 . 7}$ & 40.2 & $\mathbf{6 1 . 4}$ \\
\hline
\end{tabular}

\section{RESULTS AND ANALYSIS}

We evaluated e-Tutor using $M^{2}$ score (Dahlmeier and Ng, 2012) on the CoNLL-2014 test set, and its results are shown in Table 2. It clearly indicates that just using the synthetic pseudo-data for pre-trainingthe transformer-based model can achieve a decent performance, and e-Tutor can reach $48.0 \%$ on $F_{0.5}$. To cover more error types, we used the existing high-quality learner corpora to fine-tune the model. The results show that the model gained significant improvements on precision $(70.4 \%)$, recall $(39.6 \%)$, and $F_{0.5}(60.9 \%)$ in contrast with the pre-training process, exceeding most of published results in Tabel 2. Furthermore, after the comprehensive use of multiple error correction methods in re-editing, the precision rate of e-Tutor increased from $58.5 \%$ to $70.7 \%$.

We also compared e-Tutor with a commercial GEC application: Ginger on the CoNLL-2014 test set. The results show that the e-Tutor tool significantly improved the GEC performance and has potential to be an effective tool for ESL/EFL learners.

\section{CONCLUSION}

It is a challenging task to develop a computer-assisted language learning (CALL) tool. We designed a data augmentation method to enhance the generalizability of NMT-based GEC, which can be further improved with the specific re-editing process. In future work, we will explore a more effective data augmentation method in training GEC models. To improve GEC accuracy, we will try to build up dedicated modules to separately deal with different error types.

\footnotetext{
${ }^{1}$ https://github.com/zoncoen/python-ginger
} 


\section{ACKNOWLEDGEMENT}

This research was supported by the Humanity and Social Science Foundation of China Ministry of Education (Grant No. 15YJA740054).

\section{REFERENCES}

Brockett, C. et al., 2006, Correcting ESL Errors Using Phrasal SMT Techniques. Proceedings of the 21st International Conference on Computational Linguistics and the 44th Annual Meeting of the Association for Computational Linguistics. Stroudsburg, USA, pp 249-256.

Bryant, C. et al., 2019, The BEA-2019 Shared Task on Grammatical Error Correction. Proceedings of the 14th Workshop on Innovation Use of NLP for Building Educational Applications. Association for Computational Linguistics.

Chelba, C. et al., 2014, One billion word benchmark for measuring progress in statistical language modeling. arXiv preprint arXiv:1312.3005.

Dahlmeier, D. et al., 2013, Building a Large Annotated Corpus of Learner English: The NUS Corpus of Learner English. In Proceedings of the eighth workshop on innovative use of NLP for building educational applications, pages 22-31.

Dahlmeier, D. and Ng, H. 2012, Better Evaluation for Grammatical Error Correction. Proceedings of the 2012 Conference of the North American Chapter of the Association for Computational Linguistics: Human Language Technologies. pp 568-572.

Dale, R. and Kilgarriff, A. 2011, Helping Our Own: The HOO 2011 Pilot Shared Task. Proceedings of the 13th European Workshop on Natural Language Generation. pp 242-249.

Devlin, J. et al., 2019, BERT: Pre-training of Deep Bidirectional Transformers for Language Understanding. arXiv preprint arXiv: 1810.04805 .

Gamon, M. et al., 2008, Using Contextual Speller Techniques and Language Modeling for ESL Error Correction. In Proceedings of the Third International Joint Conference on Natural Language Processing.

Granger, S. 2014, The computer learner corpus: a versatile new source of data for SLA research.

Grundkiewicz, R. et al., 2019, Neural Grammatical Error Correction Systems with Unsupervised Pre-training on Synthetic Data. arXiv preprint arXiv:1804.05945.

Grundkiewicz, R. and Junczys-Dowmunt, M. 2018, Near Human-Level Performance in Grammatical Error Correction with Hybrid Machine Translation. arXiv preprint arXiv:1804.05945.

Heafield, K. 2011, KenLM: Faster and Smaller Language Model Queries. In Proceedings of the Sixth Workshop on Statistical Machine Translation, Association for Computational Linguistics. WMT '11, Stroudsburg, USA.

Katsumata, S. and Komachi, M. 2019. Towards Unsupervised Grammatical Error Correction using Statistical Machine Translation with Synthetic Comparable Corpus. arXiv preprint arXiv: 1907.09724.

Kiyono, S. et al., 2019, An Empirical Study of Incorporating Pseudo Data into Grammatical Error Correction. In EMNLP/IJCNLP, pp1236-1242.

Lahiri, S. 2014. Complexity of Word Collocation Networks: A Preliminary Structural Analysis. In Proceedings of the Student Research Workshop at the 14th Conference of the European Chapter of the Association for Computational Linguistics, pp 96-105.

Mizumoto, T. et al., 2011, Mining Revision Log of Language Learning SNS for Automated Japanese Error Correction of Second Language Learners. In Proceedings of 5th International Joint Conference on Natural Language Processing.

Torfi, A. et al., 2020. Natural Language Processing Advancements By Deep Learning: A Survey. arXiv preprint arXiv:2003.01200.

Warstadt, A. et al., 2019. Neural Network Acceptability Judgments. Transactions of the Association for Computational Linguistics, Vol. 7625-641.

Zhao, W. et al., 2019, Improving Grammatical Error Correction via Pre-Training a Copy-Augmented Architecture with Unlabeled Data. Proceedings of the 2019 Conference of the North American Chapter of the Association for Computational Linguistics: Human Language Technologies, Volume 1, pp 156-165. 